\title{
Colorectal Cancer Chemoprevention by Mesalazine and Its Derivatives
}

\author{
Carmine Stolfi, Francesco Pallone, and Giovanni Monteleone \\ Department of Internal Medicine, University of Rome "Tor Vergata" 1-00133, Rome, Italy \\ Correspondence should be addressed to Carmine Stolfi, carmine.stolfi@uniroma2.it \\ and Giovanni Monteleone, gi.monteleone@med.uniroma2.it
}

Received 13 March 2012; Accepted 20 April 2012

Academic Editor: Amr Amin

Copyright (C) 2012 Carmine Stolfi et al. This is an open access article distributed under the Creative Commons Attribution License, which permits unrestricted use, distribution, and reproduction in any medium, provided the original work is properly cited.

\begin{abstract}
Patients with inflammatory bowel disease (IBD) face an increased lifetime risk of developing colorectal cancer (CRC). Independent factors associated with increased risk include long disease duration, extensive colonic involvement, young age at onset of IBD, severity of inflammation, primary sclerosing cholangitis, backwash ileitis, and a family history of CRC, thus emphasising the role of intestinal inflammation as an underlying mechanism. This notion is also supported by the demonstration that the use of certain drugs used to attenuate the ongoing mucosal inflammation, such as mesalazine, seems to associate with a reduced incidence of colitis-associated CRC. In the last decade, work from many laboratories has contributed to delineate the mechanisms by which mesalazine alters CRC cell behaviour. In this paper, we review the available experimental data supporting the ability of mesalazine and its derivatives to interfere with intracellular signals involved in CRC cell growth.
\end{abstract}

\section{Introduction}

Ulcerative colitis (UC) and Crohn's disease (CD), the major forms of inflammatory bowel diseases (IBDs) in humans, are chronic inflammatory disorders of the gastrointestinal tract [1]. In UC, inflammation is restricted to the mucosa and extends proximally from the rectum to involve all or part of the colon. CD is typically a patchy, transmural, inflammatory disease that can affect the gastrointestinal tract anywhere from the mouth to the anus. Both IBDs are characterized by episodes of remission and exacerbations in which the patient experiences abdominal pain, diarrhea, blood in the stool, and systemic symptoms [1]. Patients with long-standing disease and severe inflammatory lesions involving the entire colon have increased risk of developing colorectal cancer (CRC) [2-4]. A family history of CRC, the presence of primary sclerosing cholangitis, backwash ileitis, and, in some studies, young age at onset of colitis increase further the risk of IBD-associated CRC [5-8]. CRC complicating the natural history of IBDs accounts for only 1-2\% of all cases of CRC [9]. Nonetheless, chronic colitis is among the top high-risk conditions for CRC, and IBD patients are 6 times more likely to develop CRC than the general population [10].
Unlike sporadic CRC in which the dysplastic precursor is usually the adenomatous polyp, IBD-associated dysplasia can be both polypoid and flat, localized or diffuse, and this probably reflects the fact that carcinogenesis in the inflamed colon follows a different sequence of genetic alterations than that observed in sporadic CRC [11]. The goal of CRC screening and surveillance colonoscopy in IBD population is detection of premalignant changes early enough that intervention can prevent complications of invasive cancer. Unfortunately, however, surveillance programs have not substantially prolonged survival in IBD patients, due to the technical limitations of recognizing dysplastic lesions in flat, normal-appearing mucosa [12]. This clearly suggests the need of alternative strategies of chemoprevention.

Over the last decades, the advent of sophisticated techniques of molecular biology has contributed to the identification of key steps in the process of colon carcinogenesis, thereby facilitating the development of new drugs that efficiently target malignant cells. Some of these compounds, such as nonsteroidal anti-inflammatory drugs (NSAIDs) and selective inhibitors of cyclooxygenase (COX)-2, are effective in attenuating the growth and diffusion of CRC cells $[13,14]$. 
However, their use in the chemopreventive programs of IBDrelated CRC is not justified because the administration of NSAIDs and COX2-inhibitors in IBD patients associates with high risk of disease flare-ups $[15,16]$.

Mesalazine or 5-aminosalicylic acid (5-ASA) is a drug widely used in IBDs, mainly UC, for the treatment of mild relapses and maintenance of remission. Mesalazine is structurally related to NSAIDs, but unlike these compounds, it is safe and free of serious adverse effects. Epidemiological observations indicate that mesalazine can be also chemopreventive for IBD-associated CRC $[4,17,18]$, even though one study has documented no benefit [19]. Moreover studies conducted in experimental models of carcinogenesis have shown that the drug has many targets in cancer cells and modulates multiple biological pathways that sustain CRC [20]. In this paper we review the available experimental data that demonstrate the ability of mesalazine and its derivatives to interfere with intracellular signals involved in CRC cell growth.

\section{Mesalazine Inhibits CRC Cell Growth and Survival}

The process of carcinogenesis is facilitated by a complex and dynamic interaction between genes and environmental factors that ultimately affects cell growth and survival. As a matter of fact, apoptosis progressively decreases and proliferation increases in the sequential steps from normal colonic mucosa to dysplasia and CRC. Therefore, compounds that inhibit cell proliferation and/or enhance cell apoptosis could find a place in the therapeutic armamentarium for CRC. The initial demonstration that mesalazine can block the growth and promote apoptosis of CRC cells comes from ex vivo studies in patients with colonic adenoma. ReinacherSchick et al. showed that mesalazine administered orally to patients with sporadic polyps increased the apoptotic rate and decreased proliferation of cancer cells [21]. Bus et al. demonstrated that rectal administration of mesalazine in patients with sporadic CRC enhanced apoptosis of tumor cells [22]. These observations were supported by studies in the mouse model of colitis-associated CRC, induced by administration of azoxymethane (AOM) followed by repeated oral administration of dextran sulfate sodium (DSS), which showed that mesalazine reduced the number and size of neoplasms [23, 24].

It is conceivable that the anti-neoplastic properties of mesalazine are in part linked to the ability of the drug to target mucosal immune cells and inhibit expression and/or activity of various molecules involved in colon carcinogenesis (e.g., COX-2, inducible nitric oxide synthase, reactive oxygen species and nuclear factor-kB). However, mesalazine could also regulate directly the activity of neoplastic cells. Indeed, it has been shown that mesalazine inhibits the growth and enhances the apoptosis of several CRC cell lines in a timeand dose-dependent manner [25]. The anti-mitogenic effect of mesalazine is also seen in COX-2-deficient cells raising the possibility that mesalazine inhibits CRC cell growth via both COX-2-dependent and independent mechanisms [26].
Mesalazine-induced antiproliferative effect is associated with modulation of replication checkpoints in CRC cells, which ultimately alter cell cycle progression. In this context, we have shown that increasing concentrations of mesalazine (i.e., 5-30 mM) cause a progressive accumulation of CRC cells in $S$ phase, thereby decreasing the percentage of cells in G2/M and G0/G1 [27]. Mesalazine-treated cells exhibit a reduced expression of the phosphatase CDC25A, but not CDC25B or CDC25C, and inactivation of CDK2, a target of CDC25A [27]. Since CDK2 binds to either cyclin E or cyclin $\mathrm{A}$ and regulates the G1/S transition and $\mathrm{S}$ phase progression, respectively [28], mesalazine-induced CDK2 inactivation could be responsible for cell cycle block in S-phase. The exact mechanism by which mesalazine down-regulates CDC25A expression is not yet known. It is unlikely that $\mathrm{CHK} 1$ and $\mathrm{CHK} 2$, two upstream kinases that phosphorylate and promote degradation of CDC25A are involved in the negative regulation of CDC25A, because mesalazine downregulates CDC25A, expression in CHK1- or CHK2-deficient cells [27]. By contrast, we showed that cells treated with mesalazine displayed enhanced ubiquitination and proteasome-dependent degradation of CDC25A [27]. These findings together with the demonstration that mesalazine does not affect CDC25A RNA expression strongly suggest that CDC25A is posttranscriptionally down-regulated in mesalazine-treated cells. These results are consistent with those published by Luciani and colleagues, who show that exposure of CRC cells to mesalazine causes a reversible accumulation of cells in S-phase [29]. These authors showed that such an effect was p53 independent and related with the activation of proteins involved in the ATM- and Rad3-related kinase- (ATR-) dependent S-phase checkpoint response (e.g., Chk1, RAD17) [29]. Further work by other researchers has confirmed the ability of mesalazine to interfere with cell cycle progression $[30,31]$ and shown that CRC cells can accumulate in various phases of the cell cycle in relation to the dose and time of exposure to the drug.

\section{Effects of Mesalazine on Replication Fidelity}

Both sporadic and IBD-related CRC are characterized by a very similar frequency of the two major types of genomic instability, namely, chromosomal instability (CIN) and microsatellite instability (MIN) [32]. CIN is characterized by atypical segregation of chromosomes and abnormal DNA content (aneuploidy), with consequent loss of whole (or part of) chromosomes and function of critical tumor suppressor genes (e.g., adenomatous polyposis coli, p53). MIN is characterized by an increased rate of point mutations and is dependent on defects in the mismatch repair system, a mechanism involved in repairing DNA base-pair mismatches, which occur in the normal DNA replication. During this process, frameshift mutations, called microsatellites, tend to accumulate. Microsatellites occur mainly in intronic DNA sequences thus resulting in no gene function alteration. However, when microsatellites are located in exonic gene regions, there can be a shift in the codon reading frame which results in a loss of protein function [33]. Studies performed by Gasche 
and Campregher demonstrated that mesalazine improves replication fidelity in cultured CRC cells independently of the presence of mismatch repair system $[34,35]$. The mechanism by which mesalazine inhibits the generation of frameshift mutations remains unknown, even though it could in part rely on the ability of the drug to slow down DNA replication and cell division [29], because cell cycle regulation is one of the defense mechanisms that allow cells to either repair DNA damage or eventually undergo apoptosis thus safeguarding the integrity of the genome [36].

\section{Mesalazine Inhibits Wnt $/ \beta$-Catenin Pathway and EGFR Activation}

An intriguing possibility that emerges from the available experimental data is that the antitumoral properties of mesalazine reflect the ability of the drug to target several pathways that are both early and common in colorectal carcinogenesis. The $\mathrm{Wnt} / \beta$-catenin pathway, which is constitutively activated in the majority of CRC, is one of such targets. In this pathway, Wnt binds to the transmembrane Frizzled receptor, leading to the activation of the cytoplasmic disheveled (Dsh) protein. Dsh forms a complex with the $\beta$-catenin degradation complex, which consists of the APC gene product, glycogen synthase kinase- $3 \beta$ (GSK- $3 \beta$ ), axin, and $\beta$-catenin. In the absence of Wnt, GSK- $3 \beta$ phosphorylates $\beta$-catenin, thereby promoting its ubiquitination and degradation [37]. In response to Wnt signals, $\beta$-catenin is no longer targeted for degradation, and it accumulates in the cytoplasm and subsequently translocates to the nucleus, where it associates with the transcriptional enhancers of the lymphoid enhancer-binding factor/Tcf family and stimulates the expression of genes involved in tumor progression [37]. Bos and colleagues showed that mesalazine inhibits the $\mathrm{Wnt} / \beta$-catenin pathway in APC-mutated CRC cells with intact $\beta$-catenin [38]. Mesalazine increases $\beta$-catenin phosphorylation and consequently reduces nuclear accumulation of $\beta$-catenin and expression of $\mathrm{Wnt} / \beta$-catenin target genes (e.g., cyclin D1, c-met, and c-Myc) [38]. The mechanism by which mesalazine promotes $\beta$-catenin phosphorylation remains to be ascertained, but it is known that the drug inhibits the activity of the protein phosphatase (PP)2A [38], a known regulator of the $\beta$-catenin phosphorylation status in CRC cells [39].

Another important target of mesalazine in CRC cells is epidermal growth factor receptor (EGFR), the activation of which is followed by a range of intracellular events that eventually stimulate CRC growth and survival [40]. EGFR is overexpressed not only in sporadic CRC but also in the premalignant lesions of IBD-associated carcinogenesis [41, 42 . We showed that mesalazine suppresses EGFR phosphorylation/activation in CRC cells, as a result of its ability to enhance the activity of protein tyrosine phosphatases (PTPs) that control EGFR activation [43]. In particular, mesalazine induces Src homology (SH)-PTP2 to interact with and promote dephosphorylation of EGFR [43].

\section{Mesalazine Activates PPAR- $\gamma$ in CRC Cells}

The peroxisome-proliferator-activated receptor gamma (PPAR- $\gamma$ ), a transcription factor belonging to the nuclear hormone receptor superfamily, is highly expressed in the colon where it regulates cellular proliferation, differentiation, and apoptosis [44]. PPAR- $\gamma$ activation inhibits formation of aberrant crypts foci and development of CRC in mice $[45,46]$. Rousseaux et al. showed that PPAR- $\gamma$ is a target of mesalazine in CRC cells [47]. Specifically, mesalazine enhances PPAR- $\gamma$ expression, promotes its translocation from the cytoplasm to the nucleus, and increases its interaction with vitamin D3 receptor-interacting protein-205 in CRC cells [47]. In competitive binding studies, mesalazine displaces rosiglitazone and the selective PPAR- $\gamma$ ligand GW1929 from their binding sites on the PPAR- $\gamma$ molecule [47]. In immune-deficient mice engrafted with human CRC cells, administration of mesalazine reduces the growth of xenografts, via a PPAR- $\gamma$-dependent mechanism [48]. Activation of PPAR- $\gamma$ by mesalazine is accompanied by induction of the tumor suppressor gene PTEN, activation of caspase- 8 and caspase- 3 , and diminished expression of survivin and X-linked inhibitor of apoptosis protein [49].

\section{Antineoplastic Effects of Mesalazine Derivatives}

Before generally considering mesalazine as an antitumor compound, it should be taken into consideration that the majority of preclinical studies investigating the role of this drug in CRC growth and survival have been conducted in experimental models using very high doses which are not commonly reached within the gut tissue under standard oral treatment. Therefore, the validation of novel mesalazine derivatives that exhibit similar safety profiles but enhanced anticancer activity is highly desirable.

To this end, we have recently developed several mesalazine derivatives and focused our work on 2-methoxy-5amino-N-hydroxybenzamide (we termed 2-14), since this compound displayed the more pronounced antimitogenic effect and was ten times more potent than mesalazine in blocking CRC cell growth [50]. Interestingly, 2-14 did not affect the growth of normal colonic intraepithelial lymphocytes and fibroblasts [50]. Unlike mesalazine, 214 induced endoplasmic reticulum stress (ERS) in CRC cells thereby promoting downregulation of cyclin D1 and accumulation of cells in G0/G1 phase of the cell cycle [50]. As expected, persistent block of 2-14-treated CRC cells in G0/G1 phase of cell cycle was accompanied by a caspasedependent cell death [50]. To translate these observations in vivo, we assessed the anti-neoplastic effect of 2-14 in a syngeneic CRC model, in which tumors were generated by injecting the murine CRC cell line, CT26, into Balb/c mice. Mice treated with 2-14 showed a remarkable dose-dependent decrease in tumor volume compared to control mice [50]. The anticancer effects of 2-14 was documented regardless of whether the drug was administered subcutaneously or systemically while in the same model mesalazine reduced 
the growth of tumors only when given subcutaneously [50]. We further strengthened the in vivo anti-neoplastic effect of 2-14 by using the AOM/DSS-induced colitis-associated CRC. Mice given 2-14 developed fewer smaller tumors than control mice and 2-14 treatment was not associated with significant changes in the proliferation rate of normal colonic cells [50]. More recently, we showed that 2-14 sensitized CRC cells to TNF-related apoptosis-inducing-ligand- (TRAIL-) driven death [51]. TRAIL-driven cancer cell apoptosis is mediated by ligand-dependent activation of two functional death receptors (DRs) (i.e., DR4 and DR5) [52]. Treatment of TRAIL-resistant CRC cells (i.e., HT-29 and DLD-1) with 214 but not mesalazine upregulated DR5 [51]. Moreover, 2-14 down-regulated the expression of survivin, an antiapoptotic protein that interferes with TRAIL-induced cell death in cancer cells [53]. These in vitro data were confirmed in a graft model generated by TRAIL-resistant CRC cells (i.e., CT26) in mice. In this model 2-14 synergized with TRAIL in inhibiting the in vivo growth of CT26-derived tumors [51]. In line with our results, Honeder and coworkers have recently showed that mesalazine derivatives, including $2-14$, inhibit CRC cell growth [54].

\section{Conclusion}

In the last decade extensive research has been performed to evaluate the anti-neoplastic action of mesalazine. Although definitive conclusions from these studies cannot be drawn, it seems that when used at very high doses the drug can interfere with critical steps in the process of colon carcinogenesis. Even more promising seem to be the results obtained with mesalazine derivatives. Among these, $2-14$ is more potent than mesalazine in inhibiting CRC cell growth and survival, maintains its anti-tumor activity when administered systemically, and does not affect the proliferation of normal gut cells. These observations and the fact that $2-14$ is fully synthetic and has a fairly simple structure suggest that this drug could be helpful in designing novel CRC chemoprevention programs. Further experimentation is however needed to define the pharmacokinetic properties of the compound and evaluate the potential effects of 2-14 on vital functions of the host.

\section{Acknowledgments}

This work received support from the "Fondazione Umberto di Mario", Rome, AIRC (Grant no. 9148), and Giuliani SpA, Milan, Italy.

\section{References}

[1] C. Abraham and J. H. Cho, "Inflammatory bowel disease," New England Journal of Medicine, vol. 361, no. 21, pp. 20662078, 2009.

[2] M. Rutter, B. Saunders, K. Wilkinson et al., "Severity of inflammation is a risk factor for colorectal neoplasia in ulcerative colitis," Gastroenterology, vol. 126, no. 2, pp. 451-459, 2004.

[3] R. B. Gupta, N. Harpaz, S. Itzkowitz et al., "Histologic inflammation is a risk factor for progression to colorectal neoplasia in ulcerative colitis: a cohort study," Gastroenterology, vol. 133, no. 4, pp. 1099-1105, 2007.

[4] J. A. Eaden, K. R. Abrams, and J. F. Mayberry, "The risk of colorectal cancer in ulcerative colitis: a meta-analysis," Gut, vol. 48, no. 4, pp. 526-535, 2001.

[5] J. A. Askling, P. W. Dickman, P. Karlen et al., "Family history as a risk factor for colorectal cancer in inflammatory bowel disease," Gastroenterology, vol. 120, no. 6, pp. 1356-1362, 2001.

[6] U. A. Heuschen, U. Hinz, E. H. Allemeyer et al., "Backwash ileitis is strongly associated with colorectal carcinoma in ulcerative colitis," Gastroenterology, vol. 120, no. 4, pp. 841-847, 2001.

[7] A. Ekbom, C. Helmick, M. Zack, and H. O. Adami, "Ulcerative colitis and colorectal cancer: a population-based study," New England Journal of Medicine, vol. 323, no. 18, pp. 1228-1233, 1990.

[8] J. Torres, G. P. de Chambrun, S. Itzkowitz, D. B. Sachar, and J. F. Colombel, "Review article: colorectal neoplasia in patients with primary sclerosing cholangitis and inflammatory bowel disease," Alimentary Pharmacology and Therapeutics, vol. 34, no. 5, pp. 497-508, 2011.

[9] P. M. Choi and M. P. Zelig, "Similarity of colorectal cancer of Crohn's disease and ulcerative colitis: implications for carcinogenesis and prevention," Gut, vol. 35, no. 7, pp. 950-954, 1994.

[10] J. E. Lennard-Jones, B. C. Morson, J. K. Ritchie, and C. B. Williams, "Cancer surveillance in ulcerative colitis. Experience over 15 years," The Lancet, vol. 2, no. 8342, pp. 149-152, 1983.

[11] J. M. Rhodes and B. J. Campbell, "Inflammation and colorectal cancer: IBD-associated and sporadic cancer compared," Trends in Molecular Medicine, vol. 8, no. 1, pp. 10-16, 2002.

[12] A. Ahmadi, S. Polyak, and P. V. Draganov, "Colorectal cancer surveillance in inflammatory bowel disease: the search continues," World Journal of Gastroenterology, vol. 15, no. 1, pp. 6166, 2009.

[13] C. H. Koehne and R. N. Dubois, "COX-2 inhibition and colorectal cancer," Seminars in Oncology, vol. 31, no. 7, pp. 12-21, 2004.

[14] C. V. Rao and B. S. Reddy, "NSAIDs and chemoprevention," Current Cancer Drug Targets, vol. 4, no. 1, pp. 29-42, 2004.

[15] R. Matuk, J. Crawford, M. T. Abreu, S. R. Targan, E. A. Vasiliauskas, and K. A. Papadakis, "The spectrum of gastrointestinal toxicity and effect on disease activity of selective cyclooxygenase-2 inhibitors in patients with inflammatory bowel disease," Inflammatory Bowel Diseases, vol. 10, no. 4, pp. 352-356, 2004.

[16] L. A. Feagins and B. L. Cryer, "Do non-steroidal anti-inflammatory drugs cause exacerbations of inflammatory bowel disease?" Digestive Diseases and Sciences, vol. 55, no. 2, pp. 226232, 2010.

[17] D. T. Rubin, A. LoSavio, N. Yadron, D. Huo, and S. B. Hanauer, "Aminosalicylate therapy in the prevention of dysplasia and colorectal cancer in ulcerative colitis," Clinical Gastroenterology and Hepatology, vol. 4, no. 11, pp. 1346-1350, 2006.

[18] F. S. Velayos, J. P. Terdiman, and J. M. Walsh, "Effect of 5aminosalicylate use on colorectal cancer and dysplasia risk: a systematic review and metaanalysis of observational studies," American Journal of Gastroenterology, vol. 100, no. 6, pp. 13451353, 2005.

[19] C. N. Bernstein, Z. Nugent, and J. F. Blanchard, "5-aminosalicylate is not chemoprophylactic for colorectal cancer in IBD: a population based study," American Journal of Gastroenterology, vol. 106, no. 4, pp. 731-736, 2011. 
[20] A. Lyakhovich and C. Gasche, "Systematic review: molecular chemoprevention of colorectal malignancy by mesalazine," Alimentary Pharmacology and Therapeutics, vol. 31, no. 2, pp. 202-209, 2010.

[21] A. Reinacher-Schick, F. Seidensticker, S. Petrasch et al., "Mesalazine changes apoptosis and proliferation in normal mucosa of patients with sporadic polyps of the large bowel," Endoscopy, vol. 32, no. 3, pp. 245-254, 2000.

[22] P. J. Bus, I. D. Nagtegaal, H. W. Verspaget et al., "Mesalazineinduced apoptosis of colorectal cancer: on the verge of a new chemopreventive era?" Alimentary Pharmacology and Therapeutics, vol. 13, no. 11, pp. 1397-1402, 1999.

[23] I. Ikeda, A. Tomimoto, K. Wada et al., "5-Aminosalicylic acid given in the remission stage of colitis suppresses colitisassociated cancer in a mouse colitis model," Clinical Cancer Research, vol. 13, no. 21, pp. 6527-6531, 2007.

[24] M. L. Clapper, M. A. Gary, R. A. Coudry et al., "5-aminosalicylic acid inhibits colitis-associated colorectal dysplasias in the mouse model of azoxymethane/dextran sulfate sodiuminduced colitis," Inflammatory Bowel Diseases, vol. 14, no. 10, pp. 1341-1347, 2008.

[25] A. Reinacher-Schick, A. Schoeneck, U. Graeven, I. SchwarteWaldhoff, and W. Schmiegel, "Mesalazine causes a mitotic arrest and induces caspase-dependent apoptosis in colon carcinoma cells," Carcinogenesis, vol. 24, no. 3, pp. 443-451, 2003.

[26] C. Stolfi, D. Fina, R. Caruso et al., "Cyclooxygenase-2-dependent and -independent inhibition of proliferation of colon cancer cells by 5-aminosalicylic acid," Biochemical Pharmacology, vol. 75, no. 3, pp. 668-676, 2008.

[27] C. Stolfi, D. Fina, R. Caruso et al., "Mesalazine negatively regulates CDC25A protein expression and promotes accumulation of colon cancer cells in S phase," Carcinogenesis, vol. 29, no. 6, pp. 1258-1266, 2008.

[28] G. I. Shapiro, "Cyclin-dependent kinase pathways as targets for cancer treatment," Journal of Clinical Oncology, vol. 24, no. 11, pp. 1770-1783, 2006.

[29] M. G. Luciani, C. Campregher, J. M. Fortune, T. A. Kunkel, and C. Gasche, "5-ASA affects cell cycle progression in colorectal cells by reversibly activating a replication checkpoint," Gastroenterology, vol. 132, no. 1, pp. 221-235, 2007.

[30] P. J. Koelink, M. A. C. Mieremet-Ooms, W. E. Corver et al., "5-Aminosalicylic acid interferes in the cell cycle of colorectal cancer cells and induces cell death modes," Inflammatory Bowel Diseases, vol. 16, no. 3, pp. 379-389, 2010.

[31] B. Baan, A. A. Dihal, E. Hoff et al., "5-aminosalicylic acid inhibits cell cycle progression in a phospholipase D dependent manner in colorectal cancer," Gut. In press.

[32] C. Lengauer, K. W. Kinzler, and B. Vogelstein, "Genetic instabilities in human cancers," Nature, vol. 396, no. 6712, pp. 643649, 1998.

[33] Y. Ionov, M. A. Peinado, S. Malkhosyan, D. Shibata, and M. Perucho, "Ubiquitous somatic mutations in simple repeated sequences reveal a new mechanism for colonic carcinogenesis," Nature, vol. 363, no. 6429, pp. 558-561, 1993.

[34] C. Gasche, A. Goel, L. Natarajan, and C. R. Boland, "Mesalazine improves replication fidelity in cultured colorectal cells," Cancer Research, vol. 65, no. 10, pp. 3993-3997, 2005.

[35] C. Campregher, C. Honeder, H. Chung, J. M. Carethers, and C. Gasche, "Mesalazine reduces mutations in transforming growth factor $\beta$ receptor II and activin type II receptor by improvement of replication fidelity in mononucleotide repeats," Clinical Cancer Research, vol. 16, no. 6, pp. 1950-1956, 2010.
[36] M. B. Kastan and J. Bartek, "Cell-cycle checkpoints and cancer," Nature, vol. 432, no. 7015, pp. 316-323, 2004.

[37] M. Bienz and H. Clevers, "Linking colorectal cancer to Wnt signaling," Cell, vol. 103, no. 2, pp. 311-320, 2000.

[38] C. L. Bos, S. H. Diks, J. C. Hardwick, K. V. Walburg, M. P. Peppelenbosch, and D. J. Richel, "Protein phosphatase $2 \mathrm{~A}$ is required for mesalazine-dependent inhibition of $\mathrm{Wnt} / \beta$-catenin pathway activity," Carcinogenesis, vol. 27, no. 12, pp. 2371-2382, 2006.

[39] M. J. Ratcliffe, K. Itoh, and S. Y. Sokol, "A positive role for the PP2A catalytic subunit in Wnt signal transduction," Journal of Biological Chemistry, vol. 275, no. 46, pp. 35680-35683, 2000.

[40] R. N. Jorissen, F. Walker, N. Pouliot, T. P. J. Garrett, C. W. Ward, and A. W. Burgess, "Epidermal growth factor receptor: mechanisms of activation and signalling," Experimental Cell Research, vol. 284, no. 1, pp. 31-53, 2003.

[41] M. Svrcek, J. Cosnes, E. Tiret, M. Bennis, Y. Parc, and J. F. Fléjou, "Expression of epidermal growth factor receptor (EGFR) is frequent in inflammatory bowel disease (IBD)associated intestinal cancer," Virchows Archiv, vol. 450, no. 2, pp. 243-244, 2007.

[42] J. P. Spano, C. Lagorce, D. Atlan et al., "Impact of EGFR expression on colorectal cancer patient prognosis and survival," Annals of Oncology, vol. 16, no. 1, pp. 102-108, 2005.

[43] G. Monteleone, L. Franchi, D. Fina et al., "Silencing of SHPTP2 defines a crucial role in the inactivation of epidermal growth factor receptor by 5 -aminosalicylic acid in colon cancer cells," Cell Death and Differentiation, vol. 13, no. 2, pp. 202211, 2006.

[44] M. W. Matthiessen, G. Pedersen, T. Albrektsen, S. Adamsen, J. Fleckner, and J. Brynskov, "Peroxisome proliferator-activated receptor expression and activation in normal human colonic epithelial cells and tubular adenomas," Scandinavian Journal of Gastroenterology, vol. 40, no. 2, pp. 198-205, 2005.

[45] T. Tanaka, H. Kohno, S. I. Yoshitani et al., "Ligands for peroxisome proliferator-activated receptors $\alpha$ and $\gamma$ inhibit chemically induced colitis and formation of aberrant crypt foci in rats," Cancer Research, vol. 61, no. 6, pp. 2424-2428, 2001.

[46] E. Osawa, A. Nakajima, K. Wada et al., "Peroxisome proliferator-activated receptor $\gamma$ ligands suppress colon carcinogenesis induced by azoxymethane in mice," Gastroenterology, vol. 124, no. 2, pp. 361-367, 2003.

[47] C. Rousseaux, B. Lefebvre, L. Dubuquoy et al., "Intestinal antiinflammatory effect of 5-aminosalicylic acid is dependent on peroxisome proliferator-activated receptor- $\gamma$," Journal of Experimental Medicine, vol. 201, no. 8, pp. 1205-1215, 2005.

[48] P. Desreumaux and S. Ghosh, "Review article: mode of action and delivery of 5-aminosalicylic acid-new evidence," Alimentary Pharmacology and Therapeutics, vol. 24, no. 1, pp. 2-9, 2006.

[49] M. Schwab, V. Reynders, S. Loitsch et al., "PPAR $\gamma$ is involved in mesalazine-mediated induction of apoptosis and inhibition of cell growth in colon cancer cells," Carcinogenesis, vol. 29, no. 7, pp. 1407-1414, 2008.

[50] C. Stolfi, M. Sarra, R. Caruso et al., "Inhibition of colon carcinogenesis by 2-methoxy-5-amino-N-hydroxybenzamide, a novel derivative of mesalamine," Gastroenterology, vol. 138, no. 1, pp. 221-230, 2010.

[51] C. Stolfi, R. Caruso, E. Franzè et al., "2-methoxy-5-amino-Nhydroxybenzamide, a novel derivative of mesalamine, sensitizes colon cancer cells to trail-induced apoptosis by regulating death receptor 5 and survivin expression," Molecular Cancer Therapeutics, vol. 10, no. 10, pp. 1969-1981, 2011. 
[52] A. Almasan and A. Ashkenazi, "Apo2L/TRAIL: apoptosis signaling, biology, and potential for cancer therapy," Cytokine and Growth Factor Reviews, vol. 14, no. 3-4, pp. 337-348, 2003.

[53] M. Lu, A. Strohecker, F. Chen et al., "Aspirin sensitizes cancer cells to TRAIL-induced apoptosis by reducing survivin levels," Clinical Cancer Research, vol. 14, no. 10, pp. 3168-3176, 2008.

[54] C. Honeder, C. Campregher, B. Zens, T. Scharl, and C. Gasche, "Improvement of replication fidelity by certain mesalazine derivatives," International Journal of Oncology, vol. 40, no. 5, pp. 1331-1338, 2012. 


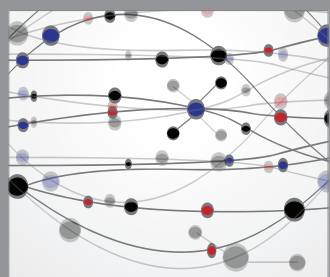

The Scientific World Journal
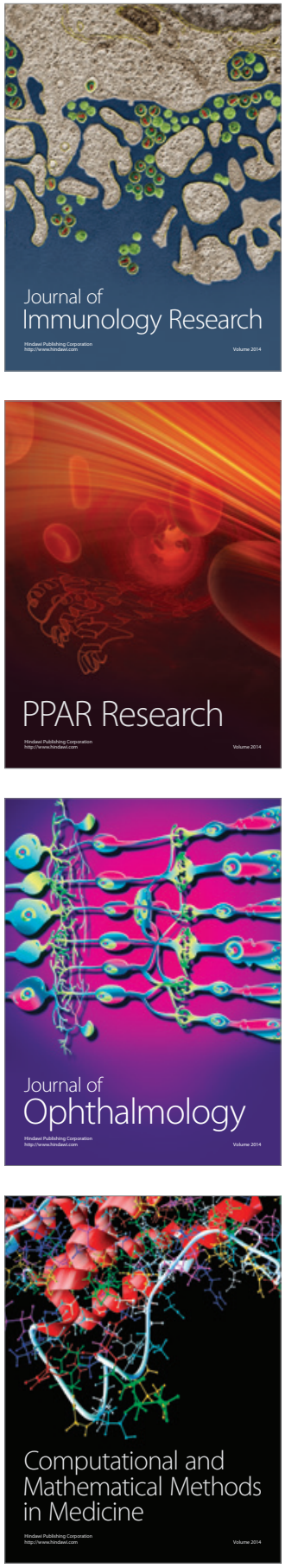

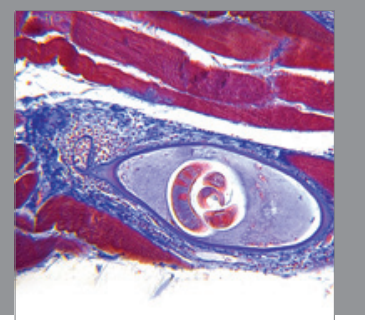

Gastroenterology

Research and Practice
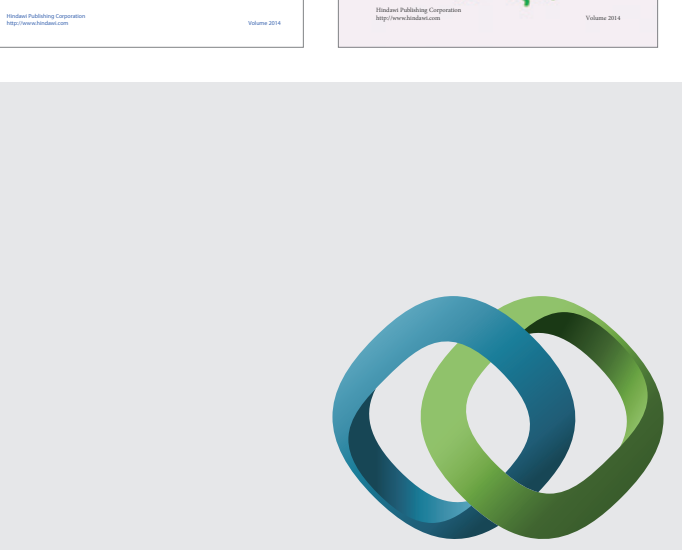

\section{Hindawi}

Submit your manuscripts at

http://www.hindawi.com
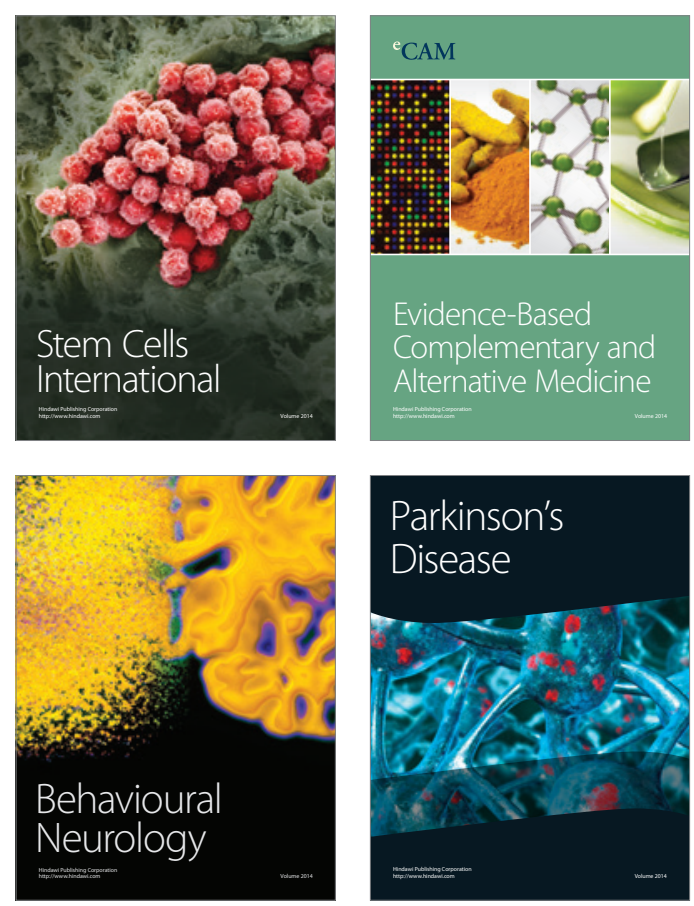

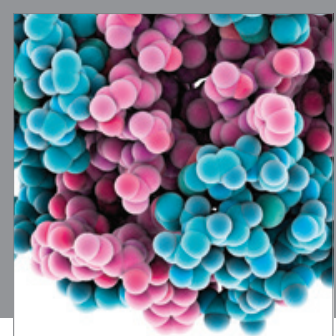

Journal of
Diabetes Research

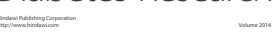

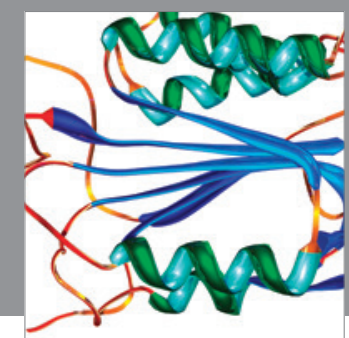

Disease Markers
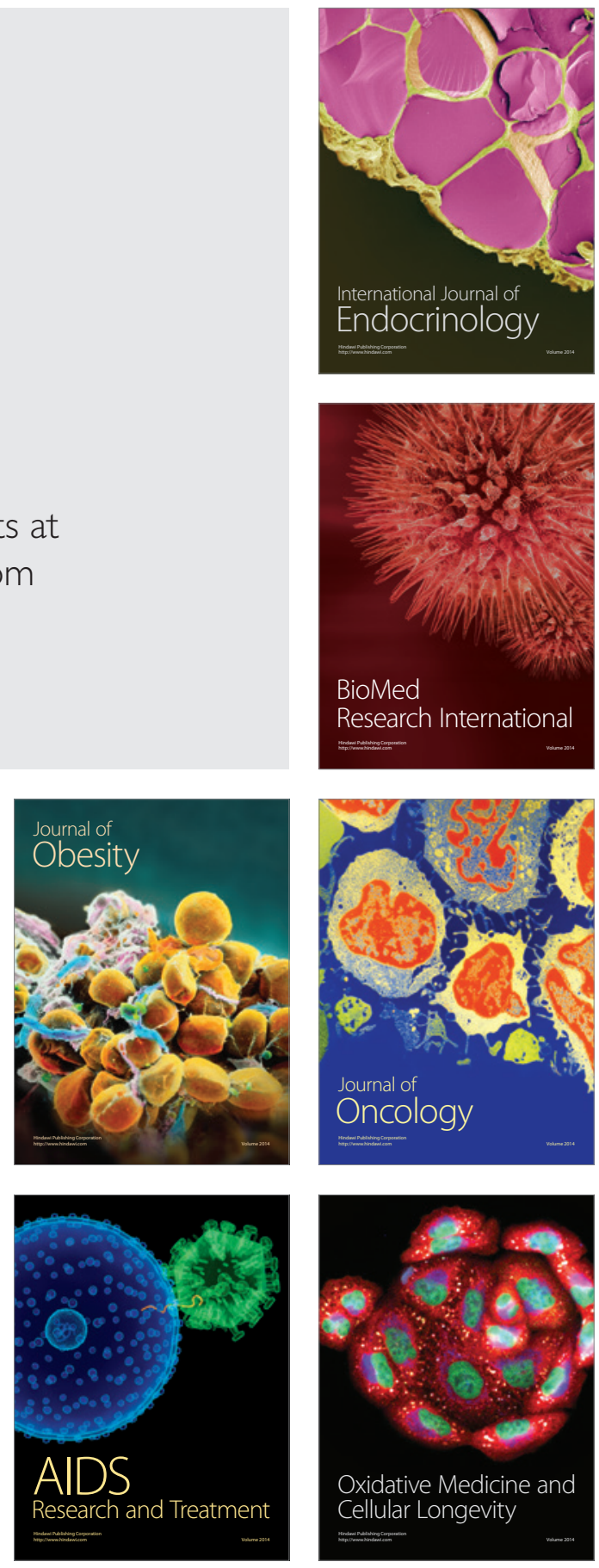